\title{
Effect of co-lipids in enhancing cationic lipid-mediated gene transfer in vitro and in vivo
}

\author{
A Fasbender ${ }^{1}, \mathrm{~J} \mathrm{Marshall}^{2}$, TO Moninger ${ }^{1}, \mathrm{~T} \mathrm{Grunst}^{1}, \mathrm{~S} \mathrm{Cheng}^{2}$ and MJ Welsh ${ }^{1}$ \\ ${ }^{1}$ Howard Hughes Medical Institute, Departments of Internal Medicine and Physiology and Biophysics, University of Iowa College of \\ Medicine, Iowa City, IA; and ${ }^{2}$ Genzyme Corporation, Framingham, MA, USA
}

\begin{abstract}
Complexes of DNA and cationic lipids are promising vectors for gene transfer. Most cationic lipid formulations contain both a cationic component and a neutral co-lipid. We found that the co-lipid could influence DNA uptake in COS1 cells, but processes subsequent to uptake were even more important in determining gene expression. We compared dioleoylphosphatidylethanolamine (DOPE) and structural analogs of DOPE combined with cationic lipids and found that DNA uptake and transgene expression did not always correlate. Transgene expression was dependent on DNA uptake into the cell, on entry of DNA into the cytoplasm, and on release of DNA from the lipid complex. We found that some co-lipids had a greater effect on DNA
\end{abstract}

uptake, whereas others had a greater effect on steps subsequent to entry. Based on those results, we tested the hypothesis that co-lipids conferring different properties could be combined to enhance gene transfer. The results showed that a combination of co-lipids had a synergistic effect on expression. We also found that structural analogs of DOPE were more effective than DOPE in enhancing gene transfer to mature human airway epithelia studied in vitro and to mouse lung studied in vivo. These data provide insight into the mechanism by which co-lipids influence cationic lipid-mediated gene transfer and show that optimization of the effects of co-lipids can enhance gene transfer both in vitro and in vivo.

Keywords: gene transfer; cationic lipids; airway epithelia; co-lipid; dioleoylphosphatidylethanolamine

\section{Introduction}

DNA complexed with cationic lipids is widely used for gene expression in the research laboratory and is being investigated for in vivo gene transfer to treat genetic and acquired diseases. Although cationic lipid vectors have a number of theoretical advantages as compared with viral vectors, they also have limitations. At present, the most formidable limitation is their low efficiency, which may be the result of several cellular and molecular barriers. ${ }^{1-6}$

Most lipid formulations used for gene transfer include both a cationic lipid and a neutral co-lipid. Although substantial attention has been paid to the cationic component, less emphasis has been placed on the neutral colipid. The cationic lipid is important because the positive charge electrostatically binds and compacts the negatively charged DNA. A net positive charge on the complex may also facilitate association with the negatively charged cell membrane. The role of the neutral co-lipid in the DNA-cationic lipid complex is less well defined.

Until recently, dioleoylphosphatidylethanolamine $(\mathrm{DOPE})^{1}$ was the neutral component in all commercially available cationic lipid preparations that contained a colipid. Previous studies have shown that DOPE is more effective than several other neutral co-lipids when combined with cationic lipids.,4,7,8 For example, dioleoylphosphatidylcholine (DOPC) was less effective

Correspondence: MJ Welsh, Howard Hughes Medical Institute, University of Iowa College of Medicine, 500 EMRB, Iowa City, IA 52242, USA Received 3 January 1997; accepted 24 March 1997 than DOPE at facilitating transfection. 3,9 There have been several proposals to explain the effect of DOPE as a colipid. It has been proposed that DOPE may promote fusion with the endosome membrane allowing release of DNA into the cytoplasm. ${ }^{8,9}$ DOPE may more effectively disrupt membranes because DOPE tends to assume a non-bilayer structure whereas DOPC tends to form a stable bilayer. ${ }^{10-13}$

The goal of this study was to examine the mechanisms by which neutral co-lipids influence cationic lipidmediated gene transfer. With that knowledge, we hoped to improve the efficiency of gene transfer. We began with an investigation of how DOPE influences transfection by measuring DNA uptake into the cell and expression of the transgene. We initially used COS-1 cells as a model system to explore the mechanisms responsible for gene transfer. Then we studied airway epithelia in vitro and in vivo, because this tissue is a target for gene transfer in the genetic disease cystic fibrosis (CF). ${ }^{14}$

\section{Results}

\section{Effect of DOPE on cationic lipid-mediated DNA uptake and expression}

To evaluate the contribution of the co-lipid to cationic lipid-mediated gene transfer to COS-1 cells, we examined the effect of including DOPE in a complex with three different cationic lipids: DC-Chol, DMRIE, and $\beta$ AEDMRIE. In each case, we evaluated DNA complexed with the cationic lipid alone, DNA complexed with the cationic lipid-DOPE liposomes, or DNA added to a mixture 
of cationic lipid and DOPE which had been formulated separately.

Because our previous work (Ref. 1 and unpublished data) had indicated that DNA uptake by cells can be a barrier to successful gene transfer, we measured both the amount of ${ }^{32} \mathrm{P}$-labeled DNA that associated with the cell and the degree of transgene expression. Figure 1 shows that incorporation of DOPE into the complex had different effects, depending on the cationic lipid; inclusion of DOPE produced a large increase in gene expression compared with DC-Chol alone, a modest increase when complexed with DMRIE, and a modest decrease compared with $\beta A E-D M R I E$ alone. These results are consistent with findings of Wheeler et al. ${ }^{15}$ When the cationic lipid and DOPE were formulated separately and then complexed with the DNA, we saw no enchancement in activity over that observed with the cationic lipid alone. This result suggests that DOPE must be incorporated into the complexing liposome for optimal activity.

Figure 1 also shows that changes in expression paralleled the effect of DOPE on DNA uptake. As described in the Materials and methods, cell-associated DNA primarily represents DNA uptake into the cell. These results suggested that DOPE may influence expression, at least in part, by determining the amount of DNA taken up into the cell.

To explore this issue further, we also tested the effect of varying the ratio of a cationic lipid (DMRIE) to DOPE in the complex. The data in Figure 2 show two things. First, inclusion of DOPE in the DNA-cationic lipid complex increased expression to a much greater extent (50100-fold) than it increased DNA uptake (approximately two-fold). Second, there was no correlation between uptake and expression. Thus, although DOPE may enhance expression in part by increasing uptake, these

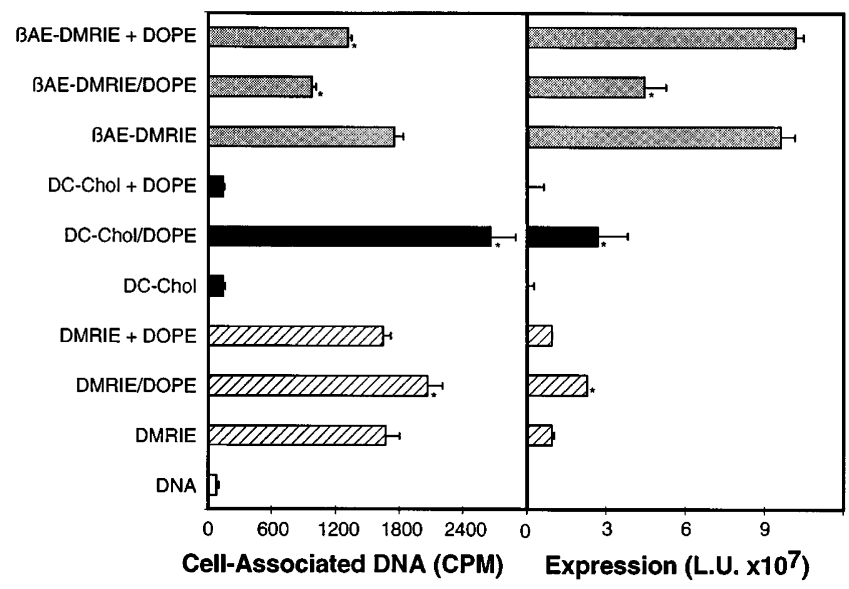

Figure 1 Effect of DOPE in combination with different cationic lipids on cell-associated DNA and transgene expression in COS-1 cells. Cells were transfected with $1 \mu \mathrm{g} p R S V L u c$ (spiked with ${ }^{32}$ P-labeled DNA) complexed to $5 \mu \mathrm{g}$ of lipid containing the cationic component alone, a cationic-DOPE mixture, or an equivalent amount of separately formulated cationic component and DOPE complexed with DNA (indicated by the + sign). Cellassociated DNA was determined immediately after the $6 \mathrm{~h}$ transfection period and expression was measured $24 \mathrm{~h}$ later. The ratio of cationic lipid to DOPE was 1:1 w/w for DMRIE/DOPE, 1:1 w/w for $\beta A E-$ DMRIE/DOPE, and 1:2.2 w/w for DC-Chol/DOPE. These ratios were chosen because we found that they produce optimal expression. $N$ was at least 6 for each condition. Asterisk indicates value different from the cationic lipid alone $(P<0.05)$.

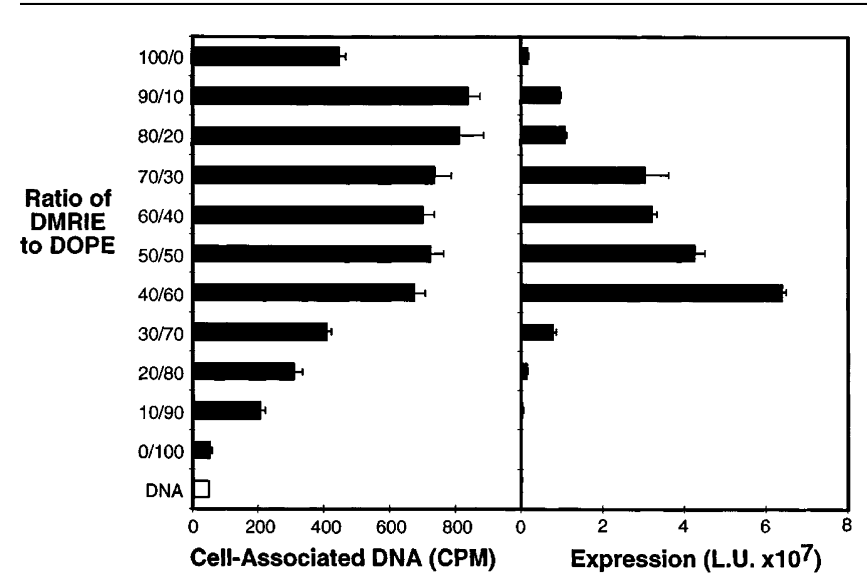

Figure 2 Effect of ratio of DMRIE to DOPE on cell-associated DNA and transgene expression. Cells were transfected with $1 \mu \mathrm{g}$ pRVLuc (spiked with ${ }^{32}$ P-labeled DNA) complexed to $5 \mu \mathrm{g}$ of DMRIE-DOPE liposomes formulated with the indicated ratios of DMRIE and DOPE. Label of the $y$-axis indicates the w/w ratio of DMRIE to DOPE in the liposomes used for complex formation. $N$ was at least 6 for each condition.

data suggest that DOPE has a more important effect at some step subsequent to DNA uptake.

Effect of DOPE analogs on DNA uptake and expression As another way to learn how DOPE influences transfection, we analyzed a series of DOPE analogs. We asked how subtle changes in the acyl chain region or in the polar head group of DOPE would affect DNA uptake and expression. For these studies, we formed complexes with DC-Chol because as shown in Figure 1, the effects of DOPE seemed to be most dramatic with this cationic lipid.

Figure 3 shows the effect of DOPE analogs in which the head group of DOPE was altered (shaded bars) or side chains at positions 1 and 2 were altered (solid bars).

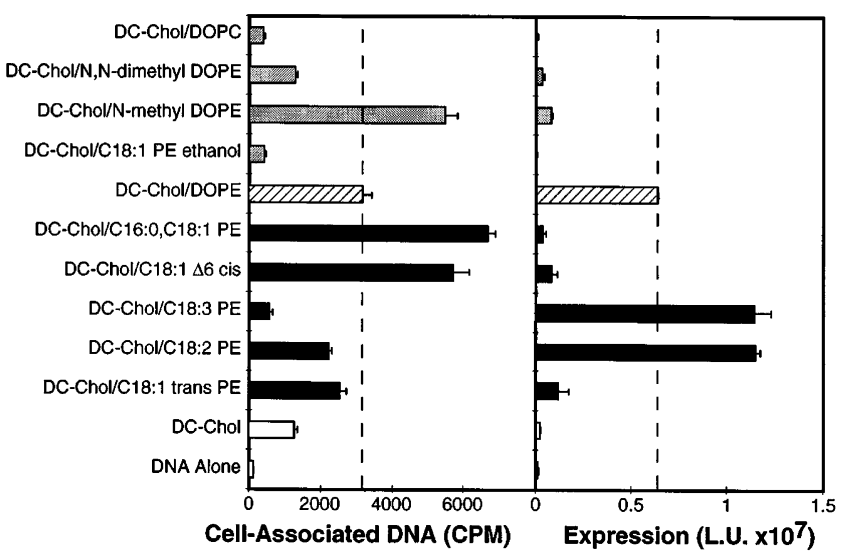

Figure 3 Effect of DC-Chol in combination with various DOPE analogs on cell-associated DNA and transgene expression. Hatched bar shows DOPE, shaded bars show analogs with altered head groups, and solid bars show analogs with altered side chains. COS-1 cells were transfected with $1 \mu \mathrm{g}$ RSVLuc (spiked with ${ }^{32} \mathrm{P}$-labeled DNA) complexed to $5 \mu \mathrm{g}$ of liposomes containing DC-Chol and the indicated DOPE analogs. The lipids were mixed in chloroform, dried to a film under a stream of argon, hydrated, and resuspended together to generate the liposomes used for complex formation. All DC-Chol:DOPE analog mixtures were at a 31:69 $w / w$ ratio in this experiment. Dashed line indicates uptake and expression with DC-Chol/DOPE for comparison. $N$ was at least 6 for each condition. 
The left panel in Figure 3 shows that the DOPE analogs had variable effects on DNA uptake relative to that obtained with DOPE (dashed line); some analogs increased and some decreased uptake. The right panel in Figure 3 shows the effect on expression. There was no correlation between the effect of the DOPE analogs on DNA uptake and on transgene expression. Note for example, that when C18:3 PE was substituted for DOPE, DNA uptake decreased, yet transgene expression increased. Conversely, when we used C16:0,C18:1 PE, DNA uptake doubled but expression fell dramatically. These data suggest that the greatest effect of DOPE and its analogs is on steps subsequent to uptake of the complex.

Mechanism of increased expression with DOPE analogs We evaluated two barriers to cationic lipid-mediated gene transfer which may have been influenced by the neutral co-lipids: escape of DNA from the endosome into the cytoplasm and release of DNA from the lipid complex. Because of their strikingly different effects on uptake and expression, for these studies we examined the effect of C18:3 PE and C16:0,C18:1 PE.

Firstly, we asked whether the neutral co-lipid would affect the amount of DNA in the cytoplasm; this would be influenced by DNA uptake into the cell and subsequent release of DNA from endosomes. We transfected COS-1 cells with increasing amounts of the DNA-lipid complex using either DC-Chol/C18:3 PE or DCChol/C16:0,C18:1 PE and a plasmid (pTM $\beta-G a l)$ in which the viral T7 promoter drives expression of $\beta$ galactosidase. We then infected the cells with a constant amount of recombinant vaccinia virus expressing the T7 RNA polymerase (vTF7-3). This system eliminates the need for plasmid entry into the nucleus by allowing cytoplasmic transcription. ${ }^{1}$ We then examined the relationship between uptake and expression. To evaluate the amount of DNA in the cytoplasm that is available for transcription, it was important to compare the two colipids at a dose of DNA that generated equivalent DNA uptake. Figure 4 shows that transfection of cells with 0.64 $\mu \mathrm{g}$ of plasmid complexed with DC-Chol/C16:0,C18:1 PE and $2.5 \mu \mathrm{g}$ of plasmid complexed with DC-Chol/C18:3
PE produced approximately similar amounts of DNA in the cell. However, cells treated with DC-Chol/C18:3 PE had 36-fold greater expression. These data suggest that the C18:3 PE co-lipid may have facilitated escape of DNA from the endosome and/or perhaps release of DNA from the lipid allowing cytoplasmic transcription. This conclusion is consistent with previous data showing that DOPE may destabilize the endosome membrane and disrupt lipid bilayers. ${ }^{8-13}$

Secondly, we used electron microscopy to compare complexes formed with the two co-lipids. Figure 5A shows that complexes formed with $\mathrm{C} 18: 3 \mathrm{PE}$ appeared to be heterogeneous and were not densely aggregated. In contrast, complexes formed with C16:0,C18:1 PE appeared as electron dense, compact particles (Figure 5B). The photomicrographs suggested that the C16:0,C18:1 PE co-lipid may have generated particles in which the complex was more tightly packed than when the C18:3 PE co-lipid was used.

Thirdly, the data in Figure 4 could be explained, in part, if the neutral co-lipid decreased the affinity with which the cationic lipid bound the DNA, allowing it to dissociate more readily. Decreased affinity would also be consistent with the electron photomicrographs in Figure 5. To evaluate this possibility we did two types of study. We reasoned that if the lipids were tightly complexed to the DNA, then DNA would be less available for labeling by nick translation. Figure 6 shows that DNA in complexes containing C16:0,C18:1 PE was less efficiently labeled than DNA in complexes containing C18:3 PE. Complexes containing DOPE were intermediate. We also examined the ability of a restriction enzyme to digest DNA that was complexed with lipid. Figure 7 shows that DNA complexed with DC-Chol/C18:3 PE was digested to a greater extent than when complexed with DCChol/C16:0,C18:1 PE. These results indicate that the colipid can influence the availability of the DNA in a complex. We interpret this to mean that the neutral co-lipid can influence the packing of DNA within the complex, and consequently the efficiency of transfection.

Finally, we used electron microscopy to examine entry of the DNA-lipid complexes following addition to the cells. In some cases we labeled the DNA with gold beads
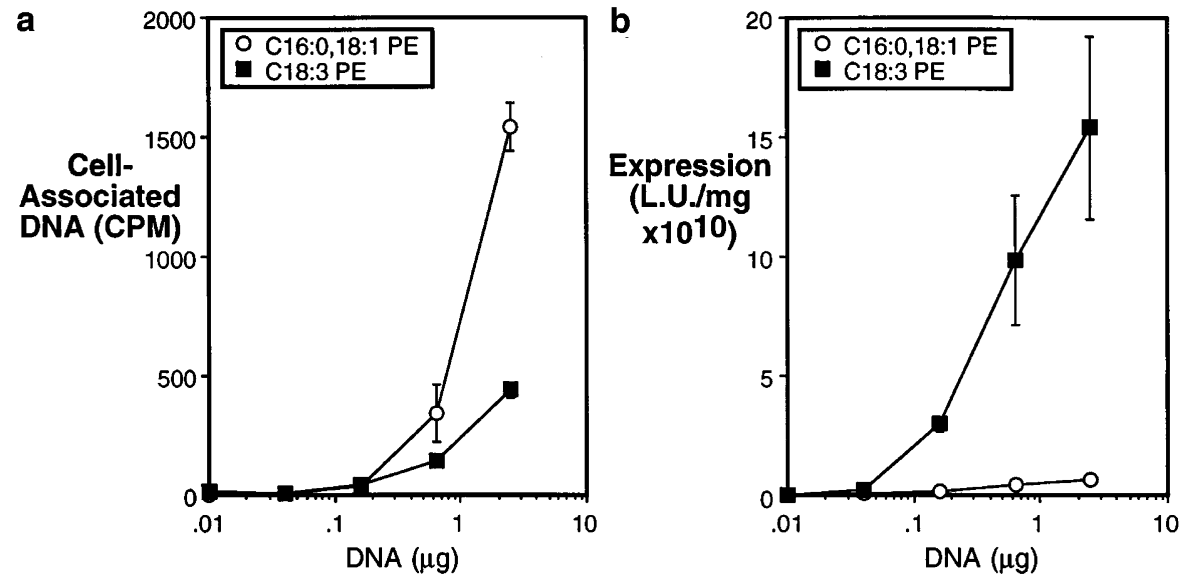

Figure 4 Cytoplasmic expression following transfection with complexes containing DC-Chol/C16:0,C18:1 PE or DC-Chol/C18:3 PE. Cells were transfected with variable amounts of $P T M-\beta G$ al complexed to $5 \times$ the weight of liposomes consisting of DC-Chol (31\% by weight) and the indicated DOPE analog (69\% by weight of the total amount of lipid). Cells were then infected with vTF7-3 (5 MOI) for 30 min. (a) Cell-associated DNA; and (b) shows $\beta$-galactosidase expression. $N$ was at least 6 at each condition. 

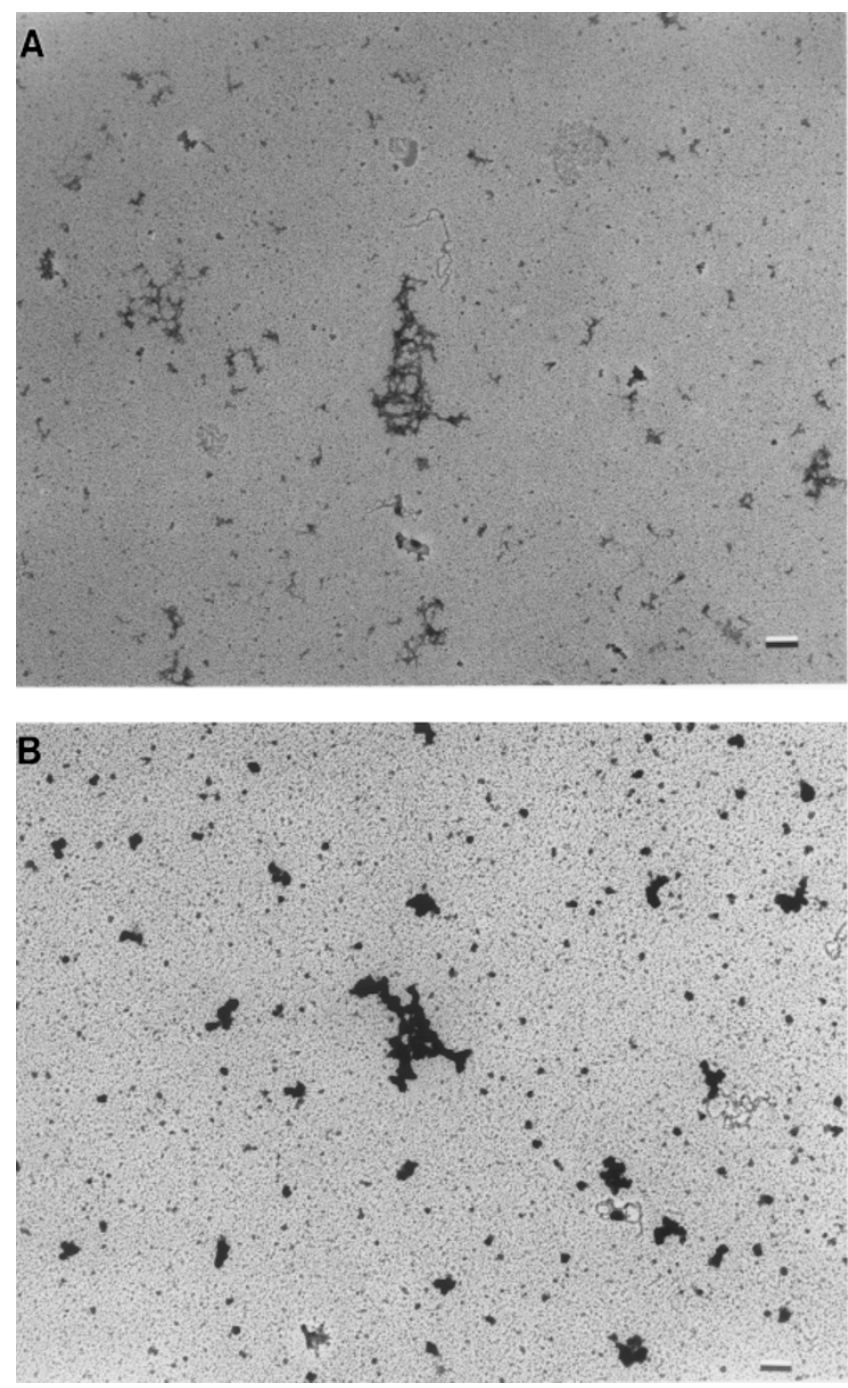

Figure 5 Electron photomicrograph of DNA complexed with: (A) DCChol/C18:3 PE or (B) DC-Chol/C16:0,C18:1 PE. Bar indicates $100 \mathrm{~nm}$.

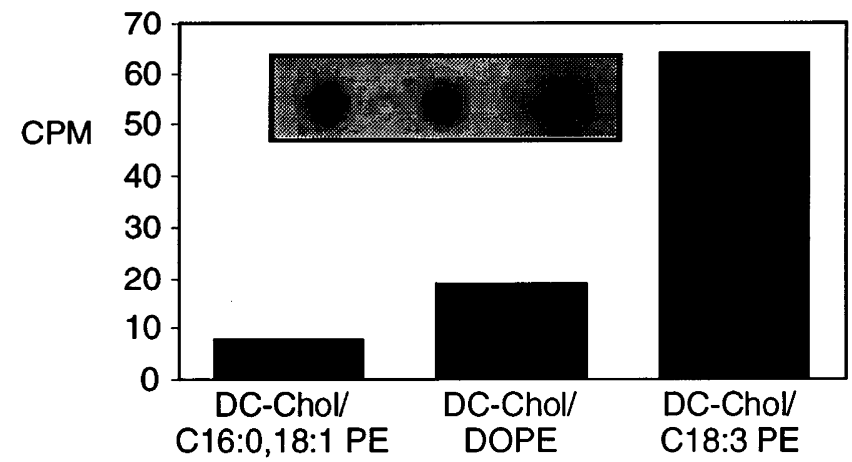

Figure 6 Nick-labeling of DNA complexed to DC-Chol/C16:0,C18:1 PE, to DC-Chol/DOPE, or to DC-Chol/C18:3 PE. Inset shows autoradiograph and bars show counts incorporated into the DNA. Experiment was repeated three times.

to be certain of the localization; however, the complexes were easily identified without labeling and we obtained similar patterns with both labeled and unlabeled DNA. Unlabeled complexes of DNA with DC-Chol/C16:0,C18:1 PE and DC-Chol/C18:3 PE are shown in Figure 8A and
Effect of co-lipids in gene transfer A Fasbender et al

$B$, respectively. The two types of complexes show very different appearances following uptake by the cells. The DNA/DC-Chol/C16:0,C18:1 PE showed closely packed extended structures in which lamellae were tight and barely visible. In contrast, the DNA/DC-Chol/C18:3 PE complexes appeared to have shorter, more fragmented, easily visualized lamellar structures. The photomicrographs suggest that complexes containing C16:0,C18:1 PE more tightly compact the DNA.

\section{Effect of combinations of DOPE analogs}

Based on our findings described above, we reasoned that we might enhance expression by combining a co-lipid that increased DNA uptake with a co-lipid that facilitated events subsequent to uptake. The hatched bars in Figure 9 show the effect of individual co-lipids. The solid bar shows that there was a synergistic effect on expression when the two co-lipids were combined during complex formation. In contrast, the shaded bars show that when only one of the neutral co-lipids was included in the complexing liposome, and the other neutral co-lipid added as a separately formed lipid dispersion, there was no synergy.

To optimize the effect of the two neutral co-lipids, we varied the ratio of $\mathrm{C} 16: 0, \mathrm{C} 18: 1 \mathrm{PE}$ to $\mathrm{C} 18: 3 \mathrm{PE}$ while keeping the DC-Chol component constant. Figure 10 shows that maximal expression occurred at a ratio of approximately 70:30 w/w. This ratio yielded intermediate levels of DNA uptake. We then used a ratio of 70:30 of C16:0,C18:1 PE to C18:3 PE and varied the ratio of DCChol to neutral lipids (Figure 11). We found that cellassociated DNA was maximal at a ratio of approximately 70:30 (DC-Chol:neutral co-lipids) and that transgene expression was maximal at a ratio of 50:50. With this formulation, expression of $\beta$-galactosidase was usually 1 log greater than that obtained with DC-Chol/DOPE.

We also found that the percentage of cells that stained positive for $\beta$-galactosidase activity by $X$-gal staining increased from $18 \pm 3 \%$ of cells with DC-Chol/DOPE to $33 \pm 4 \% \quad(P<0.05, n=3)$ with DC-Chol/C18:3 $\mathrm{PE} / \mathrm{C} 16: 0, \mathrm{C} 18: 1 \mathrm{PE}$, indicating that increased expression was not due solely to increased expression in individual cells without an increase in the number of cells transfected. Together these data indicate that gene transfer can be enhanced by taking advantage of the properties of specific neutral lipids and that a combination of neutral lipids can generate a synergistic enhancement of expression.

\section{Effect of the neutral lipid on gene transfer to human airway epithelia}

In the studies described above, we used COS- 1 cells as a model to test hypotheses about the effect of neutral lipids on gene transfer, to investigate the mechanisms involved, and to develop a system that increased expression. To test the effect of co-lipids on a more relevant cell model, we examined the effect of the co-lipid on gene transfer to human airway epithelia, a target for gene transfer in CF. ${ }^{14}$ We cultured the airway epithelia at the air-liquid interface so that they matured into a ciliated epithelium with many of the characteristics of the in vivo airway. ${ }^{16,17}$ For these studies we used a cationic lipid, GL-67, which has greater efficiency at gene transfer to airways than does DC-Chol or DMRIE (Ref. 18 and unpublished observations). In preliminary studies, we found that even 


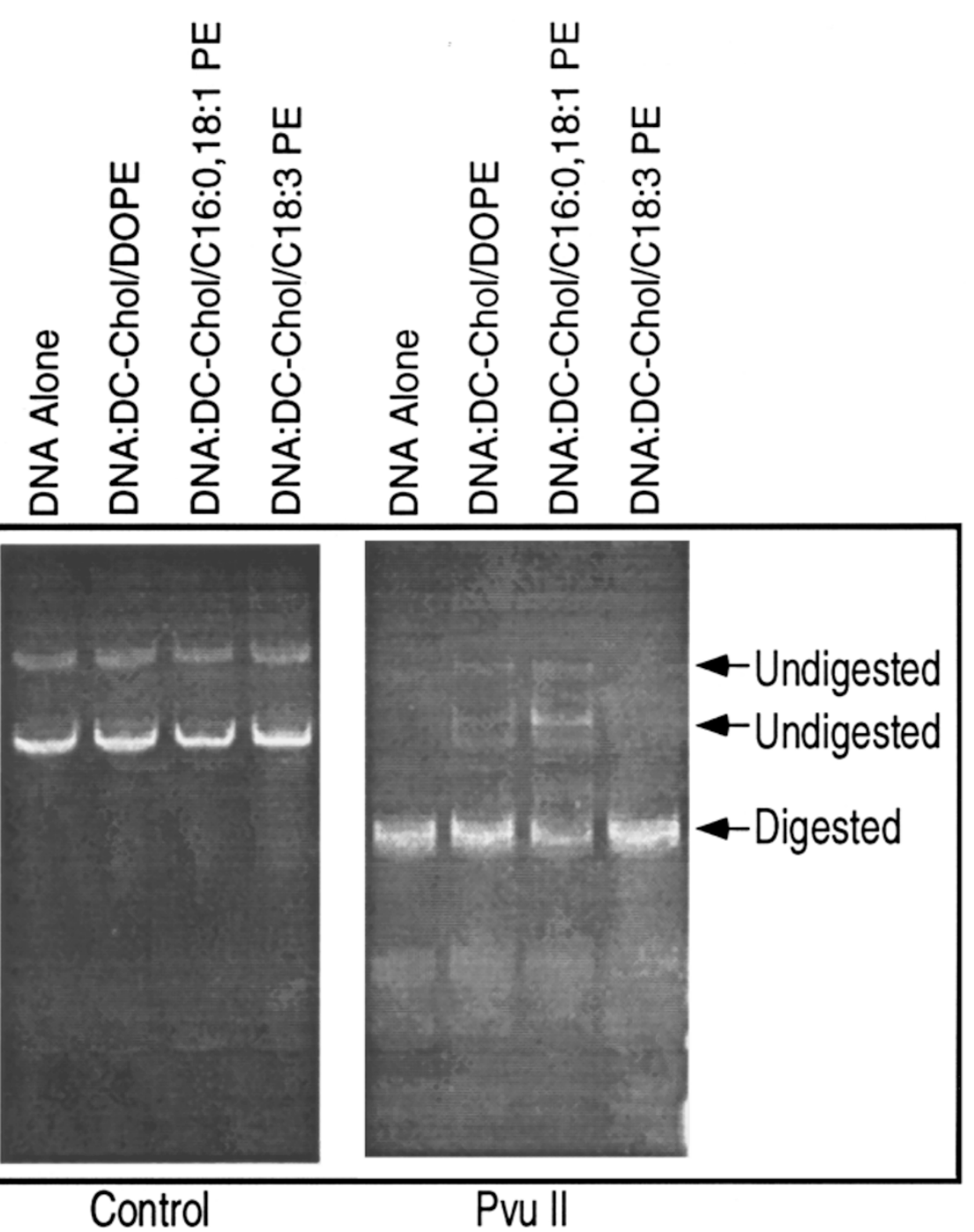

Figure 7 Restriction enzyme digestion of DNA complexed to DC-Chol/C16:0,C18:1 PE, to DC-Chol/DOPE or to DC-Chol/C18:3 PE. DNA alone and DNA complexed with the indicated lipids were treated with restriction enzyme or vehicle (control) before electrophoresis.

when combined with various co-lipids, DC-Chol was less effective than GL-67 in human airway epithelia in vitro or mouse lung in vivo (not shown). Figure 12 shows the effect of varying the neutral co-lipid on DNA uptake and transgene expression. The data show that just as we observed in COS-1 cells, the neutral co-lipid had a large effect on DNA uptake. However, specific co-lipids had different effects on uptake than they did when combined with DC-Chol and applied to COS-1 cells. The data also show that the co-lipids could have large effects on expression. In this series, inclusion of C18:2 PE in a complex with GL-67 and DNA generated the greatest expression, more than three-fold greater than with DOPE, even though DNA uptake increased by only onethird. These data indicate that the co-lipid can have important effects on transfection that appear to be subsequent to DNA uptake in a system that resembles the in vivo situation.

\section{Effect of the neutral lipid on gene transfer to mouse lung in vivo}

Based on the results in human airway epithelia, we predicted that use of C18:2 PE as the co-lipid would facilitate GL-67 mediated gene transfer in vivo. To test this, we administered DNA complexed with GL-67 plus C18:2 PE or several other neutral co-lipids. Figure 13 shows that the co-lipid had prominent effects on gene expression in the lung. As we had observed with human airway epithelia, C18:2 PE was more effective than DOPE. To optimize the effect of the co-lipid, we varied the ratio of GL67 to the neutral co-lipid C18:2 PE at a constant amount of DNA and cationic lipid. Figure 14 shows that with an approximately 1:2 molar ratio of GL-67 to C18:2 PE, we obtained the greatest transgene expression. 

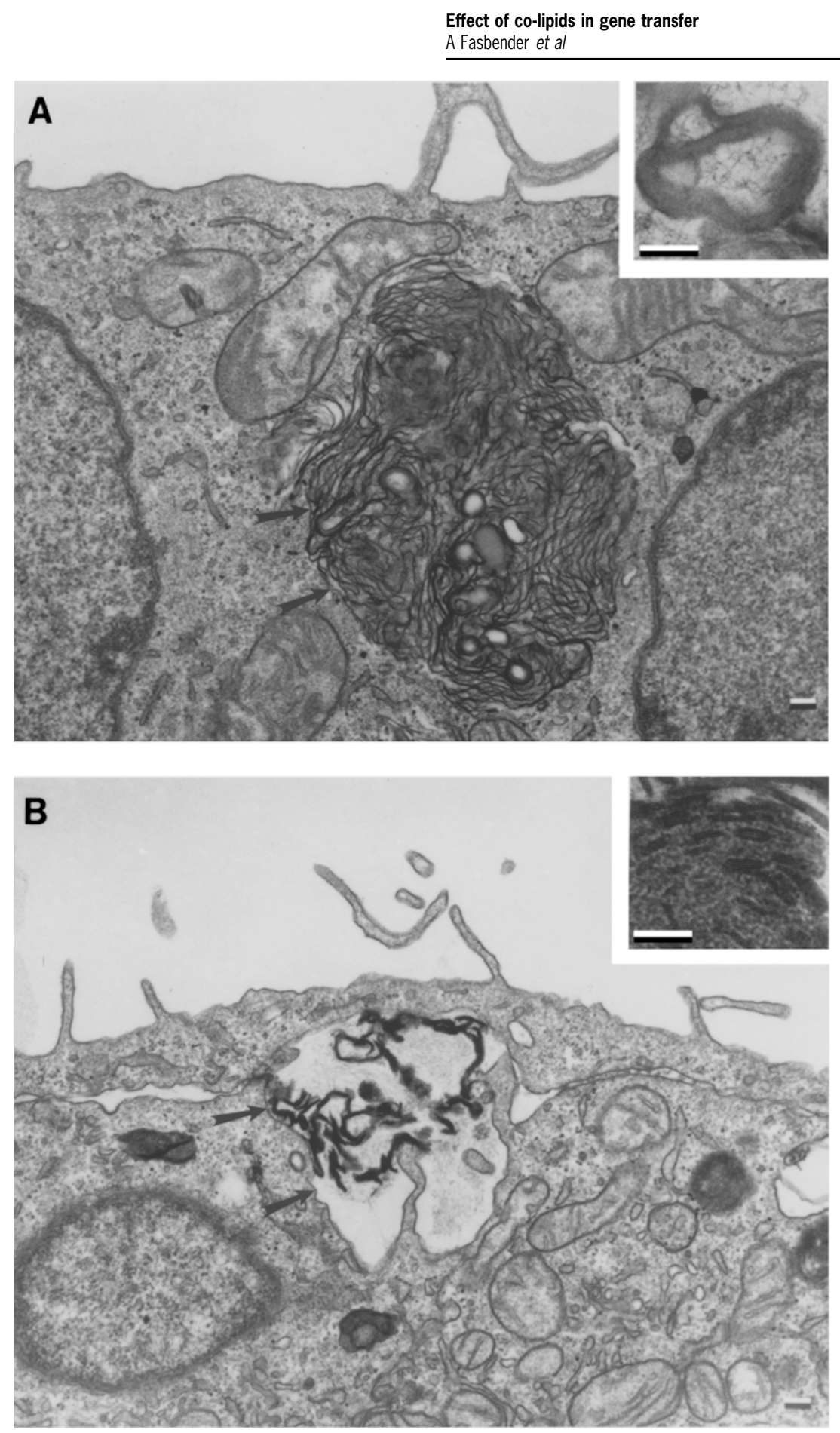

Figure 8 Electron photomicrograph of COS-1 cells treated with DNA complexed with: (A) DC-Chol/C16:0,C18:1 PE or (B) DC-Chol/C18:3 PE. Arrows indicate DNA-lipid complexes in vesicles. Insets show higher magnification of complexes. Cells received $1 \mu \mathrm{g}$ DNA complexed with $5 \mu \mathrm{g}$ lipid. Cells were transfected for $4 \mathrm{~h}$ before preparation for microscopy. Bar indicates $100 \mathrm{~nm}$.

\section{Discussion}

Our data suggest that the neutral co-lipid can play a major role in cationic lipid-mediated gene transfer. This conclusion is consistent with earlier reports that DOPE is more effective than DOPC and other neutral lipids at facilitating cationic lipid-mediated transfection. ${ }^{3,4,7,8}$ The results suggest that the neutral co-lipid can have several effects. First, it may increase DNA uptake into the cell, although this appears to have only a minimal effect on the overall efficiency of gene transfer. Second, it may facilitate escape of DNA from the endosome into the cytoplasm. Third, it may increase the ability of the DNA to dissociate from the lipid complex. Each of these mechanisms may contribute to the net amount of transgene expression. However, we also note that just as cationic lipids show different relative potencies when applied to different cells, the effect of the neutral co-lipids can vary depending on the cationic lipid and the target cell. This is apparent from an examination of the effect of the neutral co-lipids when combined with the different cationic lipids (Figures 1, 3 and 12) and also when comparing 


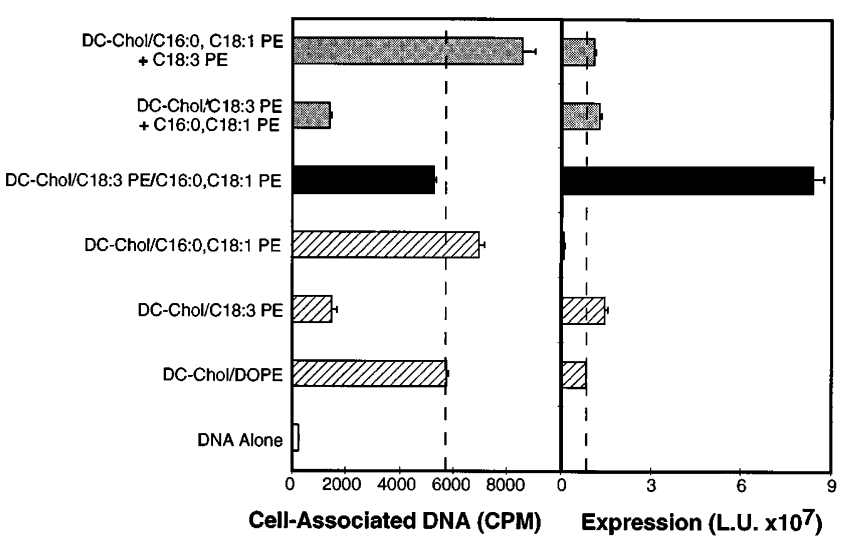

Figure 9 Effect of combining two DOPE analogs with DC-Chol on cellassociated DNA and transgene expression. Cells were transfected with $1 \mu \mathrm{g}$ of pRSVLuc (spiked with ${ }^{32}$ P-labeled DNA) complexed to $5 \mu \mathrm{g}$ of liposomes containing DC-Chol and the indicated DOPE analogs. A ' between two co-lipids indicates the lipids were mixed and resuspended together; $a$ ' $r$ ' indicates the second co-lipid was resuspended separately and then added to liposomes composed of DC-Chol and the first DOPE analog, and then complexed with DNA. Dashed line shows uptake and expression with DOPE for comparison. $N$ was at least 6 at each condition.

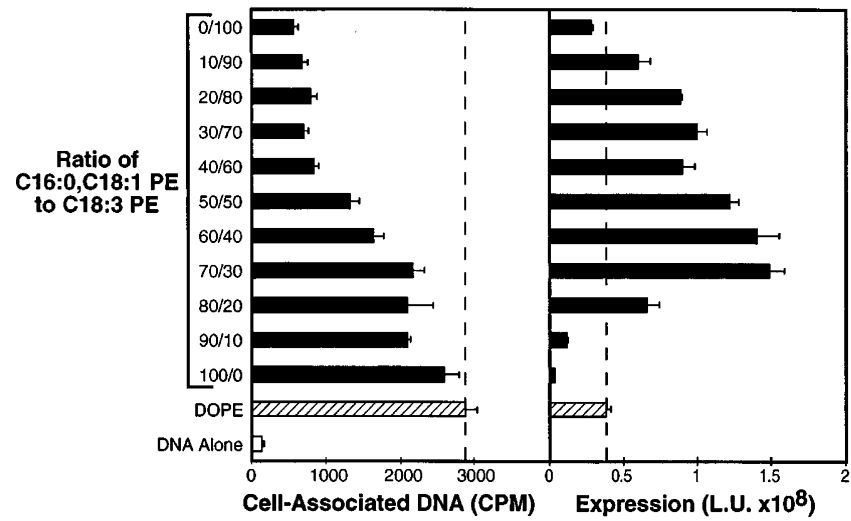

Figure 10 Effect of varying the ratio of C16:0,C18:1 PE to C18:3 PE with DC-Chol on cell-associated DNA and transgene expression. Cells were transfected with $1 \mu \mathrm{g} p R S V L u c$ (spiked with ${ }^{32}$ P-labeled DNA) complexed to $5 \mu \mathrm{g}$ of liposomes which contained a fixed amount of DC-Chol (1.6 $\mu \mathrm{g})$ together with $3.4 \mu \mathrm{g}$ of a variable ratio of C16:0, C18:1 and C18:3 $P E$. All three lipids were mixed in chloroform, dried under argon, and resuspended together to generate the liposomes used for complex formation. The $y$-axis indicates the $w / w$ percentage of the two neutral lipids in the complex. Dashed line shows uptake and expression with DOPE for comparison. $N$ was at least 6 at each condition.

expression in COS-1 cells with that in human airway epithelia and mouse lung (Figures 3, 10, 12 and 13). Reasons for cell type-specific lipid effects may include differences in cell surface composition that affect complex binding and differences in the mechanism of uptake of complex by different cell types. At present it is not possible to use a structure/activity analysis to predict the combinations of lipids that will be most effective in a specific tissue. As a result, empirical testing is still required for different applications. However, the data suggest that development of lipid-mediated gene transfer must consider the neutral co-lipid (and combinations of neutral co-lipids), in the context of the specific target cell and the specific cationic lipid.

By investigating the mechanisms by which neutral co-

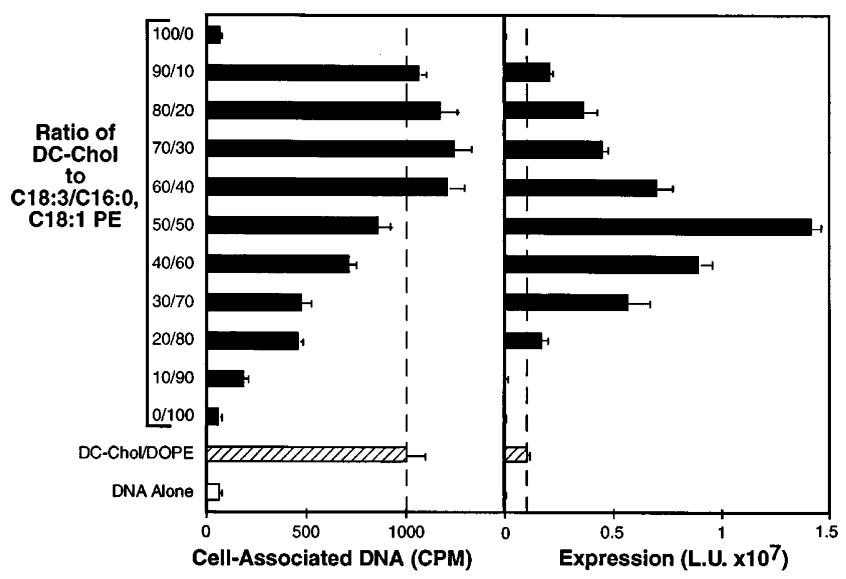

Figure 11 Effect of varying the ratio of the cationic lipid, DC-Chol, to the combination of neutral lipids, C16:0,C18:1 PE and C18:3 PE, on cellassociated DNA and transgene expression. Cells were transfected with $1 \mu \mathrm{g}$ RSSVLuc (spiked with ${ }^{32} \mathrm{P}$-DNA) complexed to $5 \mu \mathrm{g}$ of liposomes which contained a variable ratio of DC-Chol to C16:0,C18:1/C18:3 PE (the ratio of DOPE analogs was fixed at a 70:30 w/w ratio based on the results of experiments like those shown in Figure 10). All three lipids were mixed in chloroform, dried under argon, and resuspended together to generate the liposomes used for complex formation. The $y$-axis indicates the w/w percentage of the DC-Chol to the neutral lipid combination. Dashed line shows uptake and expression with DOPE for comparison. $N$ was at least 6 at each condition.

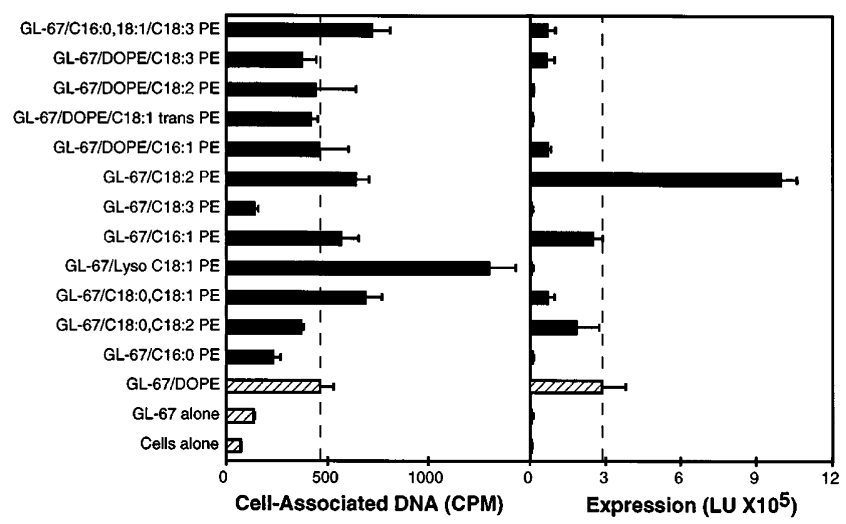

Figure 12 Effect of GL-67 in combination with various DOPE analogs on cell-associated DNA and transgene expression in primary cultures of human airway epithelia. Epithelia grown on filters at the air-liquid interface for at least 10 days were transfected with $2 \mu \mathrm{g}$ pRSVLuc (spiked with ${ }^{32}$ P-labeled DNA as described) complexed to $5.7 \mu \mathrm{g}$ of liposomes containing GL-67 and the indicated DOPE analogs. The lipids were mixed in chloroform, dried under argon, and resuspended together to generate the liposomes used for complex formation. Mixtures of GL-67 and the DOPE analogs were at a 31:69 w/w ratio in this experiment. Dashed line shows uptake and expression with DOPE for comparison. At least six epithelia were studied at each condition.

lipids affected gene transfer, we were able to combine two neutral co-lipids with different properties to produce a complex with a cationic lipid and DNA that enhanced gene transfer to an extent greater than that obtained when any single neutral co-lipid was incorporated into the complex. These results also emphasize the value of understanding the mechanisms by which various lipids produce gene transfer; the knowledge obtained from such studies can suggest approaches to improving the efficiency of gene transfer. Finally, our results show that 


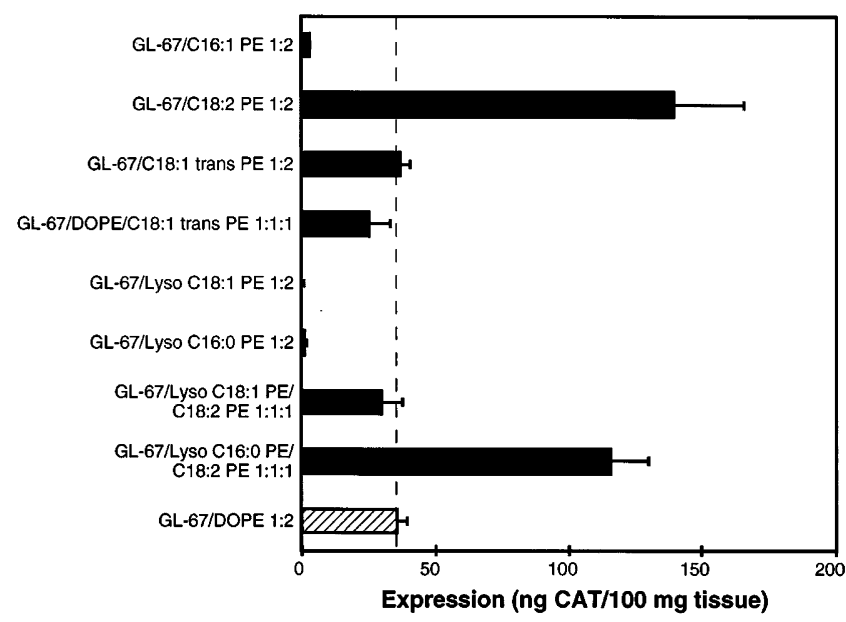

Figure 13 Effect of complexes containing DNA, GL-67, and various neutral co-lipids on gene transfer to mouse lung. Mice received $120 \mu \mathrm{g}$ of DNA complexed with $126 \mu \mathrm{g}$ of the indicated lipids. Dashed line shows uptake and expression with DOPE for comparison. At least 10 mice were studied at each condition.

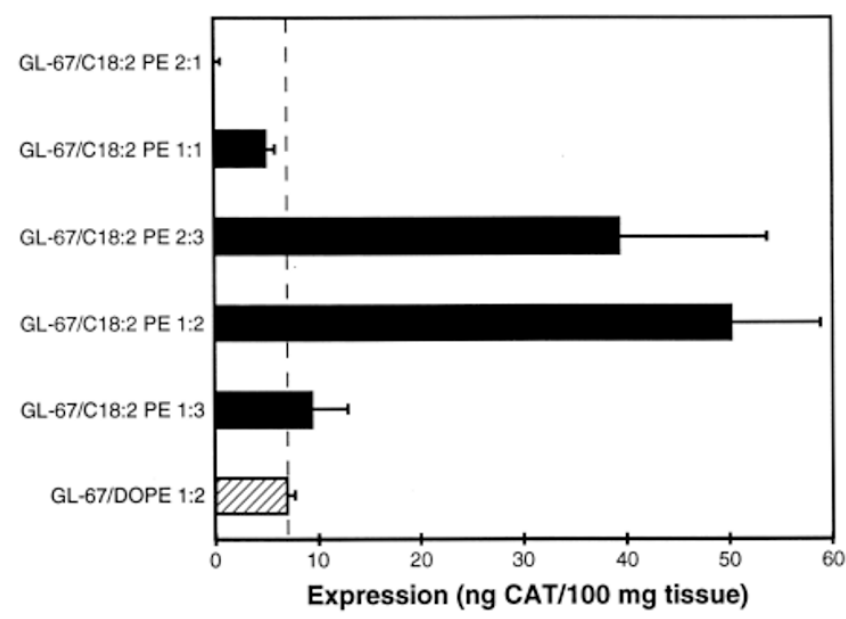

Figure 14 Effect of ratio of GL-67 to C18:2 PE on gene transfer to mouse lung. Formulations of GL-67 were prepared with respective molar ratios ranging from 2:1 to 1:3. These were compared with formulations of GL67 with DOPE. All cationic lipid:neutral lipid formulations were complexed with $p C F 1-C A T$ plasmid at a 0.6:3.6 $\mathrm{mm}$ ratio of cationic lipid to nucleotide and instilled into BALB/c mice. Dashed line shows uptake and expression with DOPE for comparison. At least 10 mice were studied at each condition. Note that the absolute levels of CAT activity were different for data shown in Figures 13 and 14. We observed variability in absolute levels of transgene expression from one experiment to another; for that reason we always compared data within an experimental group of mice.

additional manipulations of the neutral lipids can increase gene transfer when we used an in vitro model of the mature human airway and the in vivo mouse lung. These are important target tissues and models for CF. Perhaps similar modifications may improve the efficiency of gene transfer to other cells and organs that are targets for gene therapy.

\section{Materials and methods}

\section{Cells}

COS-1 cells were cultured in DMEM (high glucose) supplemented with $10 \%$ fetal calf serum, 100 units $/ \mathrm{ml}$
Effect of co-lipids in gene transfer

A Fasbender et al

penicillin, and $100 \mu \mathrm{g} / \mathrm{ml}$ streptomycin. Cells were seeded at $6 \times 10^{4}$ cells per well on to 24 -well plates $24 \mathrm{~h}$ before transfection. Cells were about $70 \%$ confluent at the time of transfection. Primary cultures of human airway epithelia were grown at the air-liquid interface and allowed to mature for at least 10-14 days before use, as previously described..$^{16,17}$

\section{Plasmids and vaccinia virus}

To assess expression in mammalian cells we used the following plasmids: pRSVLuc, containing the luciferase cDNA driven by the RSV promoter; $\mathrm{pCMV} \beta \mathrm{Gal}$, containing the cDNA for $\beta$-galactosidase driven by the CMV promoter; $\mathrm{pTM} \beta \mathrm{Gal}$, containing the cDNA for $\beta$-galactosidase driven by the T7 promoter; and pCF1-CAT expressing chloramphenicol acetyltransferase used for studies in animals. ${ }^{18}$ Plasmid DNA was purified on Qiagen columns (Qiagen, Chatsworth, CA, USA). ${ }^{32} \mathrm{P}-\mathrm{lab}-$ eled pRSVLuc was prepared by labeling $50 \mathrm{ng}$ of plasmid DNA with $50 \mu \mathrm{Ci}$ of ${ }^{32} \mathrm{P}-\mathrm{dCTP}$ (ICN Radiochemicals, Irvine, CA, USA) according to a protocol provided with the oligolabeling kit (Pharmacia Biotech, Piscataway, NJ, USA). The labeled plasmid was separated from unincorporated ${ }^{32} \mathrm{P}-\mathrm{dCTP}$ with a Sephadex G-50 Nick Column (Pharmacia Biotech) and eluted with TE buffer (10 mM Tris, 1 mм EDTA, $\mathrm{pH}=7$ ).

For some studies we used a recombinant vaccinia virus that expresses the T7 RNA polymerase (vTF7-3) and pTM- $\beta$ Gal. ${ }^{1}$ COS- 1 cells were transfected with increasing amounts of pTM- $\beta$ Gal $\left(0.005-1.3 \mu \mathrm{g}\right.$ ) (spiked with ${ }^{32} \mathrm{P}$ labeled DNA) complexed to either DC-Chol/C16:0,18:1 PE or DC-Chol/C18:3 PE (both in a 31:69 w/w molar ratio DC-Chol to PE analog). After $4 \mathrm{~h}$, the transfection solution was removed, cells were washed with Eagle's MEM, infected with vTF7-3 (5 MOI) for $30 \mathrm{~min}$, and then washed with Eagle's MEM. Half the dishes were used to measure DNA uptake and the other half were used to measure expression.

\section{Lipids}

DMRIE (N-(2-hydroxyethyl)-N,N-dimethyl-2,3-bis(tetradecyloxy)-1-propanaminium bromide) and $\beta$ AE-DMRIE (N-(2-aminoethyl)-N,N-dimethyl-2,3-bis(tetradecyloxy)1-propanaminium bromide) were gifts from Dr Phil Felgner, Vical, San Diego, CA, USA. DC-Chol was obtained from Genzyme. All co-lipids were purchased from Avanti Polar Lipids, Alabaster, AL, USA. Fresh stock solutions were used for preparing lipid formulations. Lipid purity was not checked any further. Lipid stock solutions (in chloroform) were kept at $-20^{\circ} \mathrm{C}$ under argon in the dark. The cationic component and the PE analog were mixed at $0^{\circ} \mathrm{C}$ in chloroform, dried down to a lipid film under a stream of argon, and stored at $-20^{\circ} \mathrm{C}$ until use. At the time of transfection, the lipid film was hydrated with sterile water for injection (Abbott Laboratories, Chicago, IL, USA) at $1 \mu \mathrm{g}$ total lipid per microliter water and resuspended by rapidly vortexing for $1 \mathrm{~min}$.

\section{Transfection}

Plasmid DNA and resuspended lipid were both diluted in Eagles's MEM at $15-20^{\circ} \mathrm{C}$, mixed by inversion, and allowed to incubate at room temperature for 10-15 $\mathrm{min}$ before applying to cells. The transfection mixture was left on the cells for $6 \mathrm{~h}$ (unless otherwise noted). Half the cultures were harvested for the measurement of cell- 
associated radiolabeled plasmid. Media was replaced on the other cultures, which were harvested at $24 \mathrm{~h}$ for evaluation of expression.

\section{Measurement of transgene expression}

Luciferase activity (Promega, Madison, WI, USA) and $\beta$-galactosidase (Galacto-Lite kit, Tropix, Bedford, MA, USA) were assayed with a luminometer (Monolight 2010, Analytical Luminescence Laboratory, Ann Arbor, MI, USA). Protein was measured using the Bio-Rad protein assay (Bio-Rad Laboratories, Hercules, CA, USA). Data for luciferase activity and $\beta$-galactosidase activity represent total values from all cells in one well. In each experiment, triplicate cultures were used for each condition. For X-gal staining, cells were fixed with $1.8 \%$ formaldehyde and $0.2 \%$ glutaraldehyde and then incubated for $16 \mathrm{~h}$ at $37^{\circ} \mathrm{C}$ with $0.313 \mu \mathrm{l}$ of $40 \mathrm{mg} / \mathrm{ml} \mathrm{X}$-gal (5-bromo-4-chloro-3-indolyl- $\beta$-D-galactopyranoside) in DMSO dissolved in $12.5 \mathrm{ml}$ of PBS ( $\mathrm{pH}$ 7.8). Blue staining of nuclei was evaluated by light microscopy. Results are expressed as a percentage of cells stained; at least 1000 cells were counted in each experiment.

\section{Measurement of cell-associated DNA}

For transfection $240 \mu \mathrm{g}$ of DNA was spiked with $12.5 \mathrm{ng}$ of ${ }^{32} \mathrm{P}$-labeled DNA. At the end of the transfection period, cells were washed four times with PBS $\mathrm{pH} 7.4$, then removed for scintillation counting (RACKBETA model 1209; LKB Wallac, Gaithersburg, MD, USA).

We evaluated several washing methods (including incubation of cells with phospholipase D and DNase) to determine an optimum procedure for removing noninternalized cationic lipid-DNA complex from the cells. In our hands, none was found to be more effective than the multiple PBS washing. In addition, our previous ${ }^{1}$ and current electron microscopic studies of gold-labeled DNA suggest that after thorough washing little if any DNA remains bound to the outside of the cell. These results suggest that measurement of cell-associated DNA represents primarily uptake of DNA into the cell. However, we did not evaluate the washing procedure for every formulation. Because the various DNA-lipid complexes may vary in size, shape, and physical characteristics, the efficiency of the washing procedure may not be the same for all complexes.

\section{Electron microscopy}

DNA-lipid complexes were processed for transmission electron microscopy using a negative stain/rotary shadow technique as previously described. ${ }^{1}$ To follow the cellular entry and fate of DNA, cells were transfected with DNA-lipid complexes and fixed at various times in $2.5 \%$ glutaraldehyde and processed using standard EM procedures as previously described. ${ }^{1}$

\section{Nick translation}

Nick translation was performed to examine the availability of the DNA in the DNA-lipid complex. Plasmid DNA complexed with lipid was radiolabeled by nick translation (Boehringer Mannheim, Indianapolis, IN, USA) according to the manufacturer's instructions. Lab- eled DNA was purified from the unincorporated nucleotides with a Sephadex G-50 Nick Column (Pharmacia Biotech). The amount of radiolabel in the DNA was evaluated by dot blot and quantified by radioanalytic scanning (AMBIS Systems, Include, San Diego, CA, USA).

\section{Restriction enzyme analysis}

We evaluated the availability of DNA in the DNA-lipid complex to digestion with restriction enzyme. pCMVßGal complexed with lipid was digested with PvuII for $6 \mathrm{~h}$. After digestion, the DNA-lipid complexes were dissociated with $1 \%$ SDS and then electrophoresed on a $1 \%$ agarose gel. DNA was stained with ethidium bromide and visualized with UV light.

\section{In vivo studies in mice}

GL-67 and neutral co-lipids were mixed in chloroform at the indicated molar ratios and the resultant lipid mixture was evaporated to a dry film in glass vials. In some experiments, two different neutral co-lipids were used in the same formulation, in which case the ratio of GL67:neutral lipid type 1:neutral lipid type 2 was 1:1:1. Before use, the dried lipid films were hydrated for $10 \mathrm{~min}$ with water (WFI, Baxter), vortexed for 2 min to yield a 1.2 mM suspension of GL-67. Concurrently, pCF1-CAT was diluted with WFI to $7.2 \mathrm{~mm}$ (molarity refers to the molarity of the nucleotides contained in the DNA and was calculated based on an average molecular weight per nucleotide of 330). The liposomes and pCF1-CAT DNA were warmed separately to $30^{\circ} \mathrm{C}$ for $5 \mathrm{~min}$ after which an equal volume of the liposomes was added to an equal volume of DNA and then the mixture was maintained at this temperature for a further $15 \mathrm{~min}$ to form complexes. For all experiments, $100 \mu \mathrm{l}$ of the complex at a final concentration of $0.6 \mathrm{~mm}$ GL-67 and $3.6 \mathrm{~mm}$ pCF1-CAT were administered to the lungs of anesthetized BALB/c mice as described previously. ${ }^{18}$ Complexes were administered by nasal instillation during inspiration and the animals were killed $48 \mathrm{~h}$ later. CAT expression was determined as described previously. ${ }^{18}$

\section{Acknowledgements}

We thank Pary Weber, Phil Karp, Christopher Welsh, Alaina Kehrli, Gina Hill, Beth Calease, and Theresa Mayhew for excellent assistance. We thank Dr Phil Felgner (Vical Inc, San Diego, CA, USA) for the gift of the DMRIE and $\beta A E-D M R I E$. We thank the University of Iowa Gene Transfer Vector Core (supported in part by the Roy J Carver Charitable Trust), the Central Microscopy Research Facility, and the Diabetes and Endocrine Research Center (NIH Grant DK25295) for help and support. This work was supported by a grant from the National Heart Lung and Blood Institute (HL51670), the Cystic Fibrosis Foundation, and by the Howard Hughes Medical Institute (HHMI). MJW is an Investigator of the HHMI. 


\section{References}

1 Zabner J et al. Cellular and molecular barriers to gene transfer by a cationic lipid. J Biol Chem 1995; 270: 18997-19007.

2 Friend DS, Papahadjopoulos D, Debs RJ. Endocytosis and intracellular processing accompanying transfection mediated by cationic liposomes. Biochim Biophys Acta 1996; 1278: 41-50.

3 Wrobel I, Collins D. Fusion of cationic liposomes with mammalian cells occurs after endocytosis. Biochim Biophys Acta 1995; 1235: 296-304.

4 Gao X, Huang L. Cationic liposome-mediated gene transfer. Gene Therapy 1995; 2: 710-722.

5 Legendre JY, Szoka FCJ. Delivery of plasmid DNA into mammalian cell lines using $\mathrm{pH}$-sensitive liposomes: comparison with cationic liposomes. Pharm Res 1992; 9: 1235-1242.

6 Meyer KB et al. Intratracheal gene delivery to the mouse airway: characterization of plasmid DNA expression and pharmacokinetics. Gene Therapy 1995; 2: 450-460.

7 Leventis R, Silvuis JR. Interactions of mammalian cells with lipid dispersions containing novel metabolizable cationic amphiphiles. Biochim Biophys Acta 1990; 1023: 124-132.

8 Zhou X, Huang L. DNA transfection mediated by cationic liposomes containing lipopolylysine: characterization and mechanism of action. Biochim Biophys Acta 1994; 1189: 195-203.

9 Farhood H, Serbina N, Huang L. The role of dioleoyl phosphatidylethanolamine in cationic liposome mediated gene transfer. Biochim Biophys Acta 1995; 1235: 289-295.

10 Wimley WC, Thompson TE. Phosphatidylethanolamine enhances the concentration-dependent exchange of phospholipids between bilayers. Biochemistry 1991; 30: 4200-4204.
11 Ellens H, Bentz J, Szoka FC. Destabilization of phosphatidylethanolamine liposomes at the hexagonal phase transition temperature. Biochemistry 1986; 25: 285-294.

12 Brown PM et al. Role of head group structure in the phase behavior of amino phospholipids. 2. Lamellar and nonlamellar phases of unsaturated phosphatidylethanolamine analogues. Biochemistry 1986; 25: 4259-4267.

13 Allen TM, Hong K, Papahadjopoulos D. Membrane contact, fusion, and hexagonal (HII) transitions in phosphatidylethanolamine liposomes. Biochemistry 1990; 29: 2976-2985.

14 Welsh MJ, Tsui L-C, Boat TF, Beaudet AL. Cystic fibrosis. In: Scriver CR, Beaudet AL, Sly WS et al (eds). The Metabolic and Molecular Basis of Inherited Disease. McGraw-Hill: New York, 1995, pp 3799-3876.

15 Wheeler CJ et al. Converting an alcohol to an amine in a cationic lipid dramatically alters the co-lipid requirement, cellular transfection activity and the ultrastructure of DNA-cytofectin complexes. Biochim Biophys Acta 1996; 1280: 1-11.

16 Zabner J, Zeiher BG, Friedman E, Welsh MJ. Adenovirusmediated gene transfer to ciliated airway epithelia requires prolonged incubation time. J Virol 1996; 70: 6994-7003.

17 Smith JJ, Travis SM, Greenberg EP, Welsh MJ. Cystic fibrosis airway epithelia fail to kill bacteria because of abnormal airway surface fluid. Cell 1996; 85: 229-236.

18 Lee ER et al. Detailed analysis of structures and formulations of cationic lipids for efficient gene transfer to the lung. Hum Gene Ther 1996; 7: 1701-1717. 